\title{
RepuCoin: Your Reputation is Your Power
}

\author{
Jiangshan Yu, David Kozhaya, Jeremie Decouchant, and Paulo Esteves-Verissimo, Fellow, IEEE
}

\begin{abstract}
Existing proof-of-work cryptocurrencies cannot tolerate attackers controlling more than $50 \%$ of the network's computing power at any time, but assume that such a condition happening is "unlikely". However, recent attack sophistication, e.g., where attackers can rent mining capacity to obtain a majority of computing power temporarily, render this assumption unrealistic. This paper proposes RepuCoin, the first system to provide guarantees even when more than $50 \%$ of the system's computing power is temporarily dominated by an attacker. RepuCoin physically limits the rate of voting power growth of the entire system. In particular, RepuCoin defines a miner's power by its 'reputation', as a function of its work integrated over the time of the entire blockchain, rather than through instantaneous computing power, which can be obtained relatively quickly and/or temporarily. As an example, after a single year of operation, RepuCoin can tolerate attacks compromising $51 \%$ of the network's computing resources, even if such power stays maliciously seized for almost a whole year. Moreover, RepuCoin provides better resilience to known attacks, compared to existing proof-of-work systems, while achieving a high throughput of 10000 transactions per second (TPS).
\end{abstract}

Index Terms-Blockchain, cryptocurrency, fault tolerance, consensus.

\section{INTRODUCTION}

B ITCOIN [1] is the most successful decentralized cryptocurrency to date. However, despite its enormous commercial success, many weaknesses have been associated with Bitcoin, including weak consistency, low transaction throughput and vulnerabilities to attacks, such as double spending attacks [2,3], eclipse attacks [4, 5], selfish-mining attacks [6, 7], and flash attacks [8].

Several promising existing solutions [9-14] targeted the low throughput problem of Bitcoin. Nevertheless, these solutions either provide only probabilistic guarantees about transactions (weak consistency) [13] or can provide strong consistency but suffer from liveness problems even when an attacker has a relatively small computing power [15]. Moreover, the resilience of such solutions against attacks, such as selfish mining attacks where an attacker has more than $25 \%$ of the computing power $[3,6,7,16]$, remains unsatisfactory. In addition, all existing contemporary proofof-work (PoW) based variants of Bitcoin (e.g. [13, 15, 17, 18]) rely on the assumption that an attacker cannot have more than $33 \%$ or $50 \%$ of computing power at any time. However, with the sophistication of attacks mounted on Bitcoin, e.g., flash attacks (a.k.a. bribery attacks), where an attacker can obtain a temporary majority ( $>50 \%$ ) of computing power by renting mining capacity [8], all these systems would fail. In brief, existing solutions that address the weaknesses associated with Bitcoin still suffer from significant shortcomings.

This paper addresses these shortcomings - liveness of current high-throughput solutions, and vulnerability to attacks such as selfish mining and flash attacks. In particular, we propose RepuCoin, the first system that can prevent attacks against an attacker who may possess more than $50 \%$ computing power of the entire network temporarily (e.g., a few weeks or even months). Our proof-of-concept implementation shows that while providing better security guarantees than predecessor protocols, RepuCoin also ensures a very high throughput (10000 transactions per second). In practice, Visa confirms a transaction within seconds, and processes $1.7 \mathrm{k}$ TPS on average [19]. This shows that RepuCoin satisfies the required throughput of real world applications.

Design principle. Our system addresses the aforementioned challenges by defining a new design principle, called proof-of-reputation. Proof-of-reputation is based on proof-ofwork, but with two fundamental improvements.

First, under proof-of-reputation a miner's decision power (i.e., the voting power for reaching consensus in the system) is given by its reputation. A miner's reputation is not measured by what we call the miner's 'instantaneous' power, i.e., the miner's computing power in a short time range, as in classic PoW. Instead, the reputation is computed based on both the total amount of valid work a miner has contributed to the system and the regularity of that work, over the entire period of time during which the system has been active. We call this the miner's 'integrated power'. So, when an attacker joins the system at time $t$, even if it has a very strong mining ability that is, high computational (i.e., instantaneous) power, it would have no integrated power at time $t$, or even shortly after, as it did not contribute to the system before $t$.

Second, when a miner deviates from the system specifications, RepuCoin lowers the miner's reputation, and hence its integrated power, in consequence of this negative contribution. This prevents a powerful malicious miner from attacking the system repeatedly without significant consequences. In contrast, classic PoW systems either do not support any feature for punishing miners that do not abide by system specifications, or they punish these miners by merely revoking their rewards - this does not prevent them from attacking the system again immediately after.

RepuCoin provides deterministic guarantees on transactions by employing a reputation-based weighted voting consensus. Consensus is carried by a group formed of the top reputable miners. Every member of that group has a weight associated to its vote. The weight of a member's vote is the percentage of that member's reputation w.r.t. the entire group's reputation. Such weights ensure that one's voting 
power depends, not on the sheer instantaneous (computing) power - which is the enabler of flash attacks - but on the integrated power, which both takes time to build, and is built on the miner's honesty and historical performance. In fact, quantifying a miner's voting power based on its performance in the entire blockchain highlights the selfstabilizing characteristics of our approach: qualitatively, to acquire power in RepuCoin a miner is urged to exhibit normal, honest behavior; quantitatively, the speed with which a miner can gain power is dictated by the regularity and amount of that miner's contributions in the entire blockchain.

To illustrate the robustness of the design choices underlying RepuCoin, we present our analysis of the security provided by the mechanisms used in RepuCoin. In particular, we show that achieving the safety and liveness correctness conditions of the reputation-based weighted voting consensus protocol is physically guaranteed by the growth rate of the proof-of-reputation function, and the rate of decision power growth of the entire system is bounded. In addition, we present experiments exemplifying concrete values for the decision power growth in several situations, showing that the network achieves very high stochastic robustness against attacks on its liveness or safety. For instance, we demonstrate that after a single year of operation, RepuCoin is resilient to all attacks that compromise 26\%, 33\%, and 51\% of the networks computing resources, even if such power stays maliciously confiscated for almost 100 years, 2 years, and 1 year respectively. Also, in the same setting, even if an attacker can afford to seize a huge computational power for a specific period, due to the cost of such attacks (e.g., $90 \%$ for up to 3 months), it will not break RepuCoin. Moreover, we provide an analysis of the non-rationality of infiltration attacks, with a comparison of the cost of attacking different systems. Furthermore, we provide in detail how RepuCoin prevents known attacks.

\section{Related Work}

Consensus is the key component and backbone of Blockchains, which use two main types of consensus mechanisms, namely proof-of- $X$ based consensus, and Byzantinefault tolerant (BFT) consensus. The former is generally permissionless, where anyone can join and leave the potentially large consensus group; and the latter is permissioned, where the set of participants running consensus is small and predefined. Due to space limitations, we give a brief review here, and refer readers to our report [20], and the associated works for more details.

Proof-of- $X$ based consensus has gained much interest since the use of Proof-of-work in Bitcoin, already described in the introduction. Proof-of-stake, which was first discussed in a Bitcoin community forum [21], has been proposed to use virtual voting to provide quicker transaction confirmation. Proof-of-space (a.k.a. proof-of-capacity) [22, 23] has been proposed to use physical storage resources to replace computing power in the proof-of-work mechanism. Proof-of-coin-age [24] shares a similar concept as proofof-stake, as participants also perform virtual "mining" by demonstrating possession of a quantity of currency. Proofof-activity [25] puts every coin owner into a mining lottery; periodically, winners are randomly determined by transactions. A winner is expected to respond with a signed message within a small time interval to claim its award. Proofof-elapsed time [26], proposed by Intel and implemented as a Hyperledger project, uses Intel SGX enabled CPUs to do virtual voting through a random sleeping time to replace the proof-of-work mechanism. Proof-of-membership system in ByzCoin [15] creates a consensus group formed by recent PoW-based block creators, and this group runs a $\mathrm{BFT}$ protocol for reaching consensus. However, it is shown that ByzCoin still suffers from selfish mining attack [15], and that it can permanently lose liveness during reconfiguration, if too many miners disappear in a short time [27] or can repeatedly lose liveness temporarily [15]. Unfortunately, none of the permissionless consensus protocols described is able to provide a meaningful security guarantee when an attacker is able to control a majority of mining power.

Notable state of the art of BFT protocols include PBFT [28], MinBFT/MinZyzzyzva [29], or ReBFT [30]. PBFT [28] proposes the first Byzantine fault-tolerant algorithm that has an acceptable performance in practice. MinBFT/MinZyzzyva [29] are hybrid protocols presenting non-speculative and speculative BFT algorithms, which are efficient and only require $2 f+1$ replicas (rather than $3 f+1$ ) in total to tolerate a maximum number $f$ of faulty players, thanks to the use of a trusted monotonic counter associating sequence numbers to each operation. ReBFT [30] is an approach that relies on a passive-replication paradigm to minimize the number of non-faulty replicas that participate in system operations in the absence of faults.

\section{System AND ThREAT MOdEL}

\subsection{System Context}

RepuCoin makes use of two types of blocks, namely keyblock and microblock. Keyblocks are created through PoW, as a means to elect a leader, and they do not contain any transaction. Microblocks are proposed by a randomly selected leader to record transactions into the blockchain.

All participants of the system, no matter whether they are miners or mobile clients, learn about new transactions and blocks in the same way as in BitCoin and other blockchains, through a peer-to-peer protocol.

In RepuCoin, we verify and commit microblocks, as well as decide on the keyblocks to be added to the blockchain, using Byzantine fault tolerance protocols (e.g., $[28,30]$ ) with minor modifications, as presented in $\S 4.4$. Such form of agreement prevents a malicious leader from double spending a coin, and resolves potential forks resulting from simultaneously mined keyblocks. According to Byzantine quorums theory [31], in order to reach an agreement, classic BFT protocols require votes from at least $2 f+1$ nodes $^{1}$, to prevent an adversary controlling $f$ nodes from breaking the protocol. In practice, however, an open BFT-based system cannot guarantee that an attacker will never be able to control more than $f$ nodes. To enforce the assumption that no more than $f$ Byzantine nodes are ever involved in a consensus, we

1. Hybrid BFT protocols with trusted hardware components, such as MinBFT [29], only require votes from $f+1$ nodes. 
introduce novel mechanisms to make it infeasible, in practice, for an attacker to seize $f$ nodes within the consensus group, as detailed below.

\subsection{System Model}

RepuCoin is a system composed of a non-predetermined number of nodes, called miners. Each miner has a reputation score, which determines that miner's ability of obtaining rewards. A miner's reputation score is based on the correctness of its behavior and its regularity in adding blocks to the existing chain, hence correlated with the miner's computing power. RepuCoin considers a network that is untrustworthy and unreliable. In addition, we assume the network has partial synchrony [32].

To address the above-mentioned uncertainty (Section 3.1) in the definition of the consensus quorums and outcomes for open BFT-based protocols, RepuCoin resorts to two main techniques. First, we rely on the notion of having a consensus group, i.e., a subset of the miners denoted by $\mathbb{X}$ capable of controlling the operations of RepuCoin, namely running the consensus protocol. Second, the voting rules in the underlying consensus schemes are not nominal, but based on a novel reputation-based weighted voting. To this end, we refine the definition of quorums as follows: reaching agreement not only requires votes from $2 f+1$ nodes, but also demands that these nodes collectively have more than $\frac{2}{3}$ of the cumulative reputation of the consensus group.

The consensus group size is defined as the minimum number of miners with enough decision power (i.e., cumulative reputation) to ensure safe and live control of the system, given our quorum definition. Therefore, the consensus group members are obviously the miners with the highest reputation scores. This stratagem has two virtuous effects. First, RepuCoin's safety is guaranteed by consensus, which can be viewed as a deterministic control orchestrated by a set of miners with overwhelming cumulative reputation. By definition, such reputation itself gives an expectation of the correct behavior of these miners. Second, openess and fairness of RepuCoin relies on $\mathbb{X}$ being parametric and agnostic of identity of network members, as we show in Section 4.2. At configuration time, the size of $\mathbb{X}$ is calculated by meeting a target percentage of the overall decision power, and so it can be large or small, depending on the mining pool composition, but not pre-determined. On the other hand, as miners gain or lose reputation, they can (by merit or demerit) enter and leave the consensus group.

\subsection{Threat Model}

We consider a malicious (a.k.a. Byzantine) adversary, who can arbitrarily delay, drop, re-order, insert, or modify messages. We also consider collusions of an arbitrary number of miners, to model a malicious real organization capable of deploying a significant number of virtual miners under its direct dependence. We assume the security of the used cryptographic primitives, including a secure hash function and a secure signature scheme.

Such adversary can potentially control as many miners as it wishes, and coordinate them in real time with no delay. In consequence, the consensus group can be infiltrated by adversaries. However, we assume that the adversary has the ability to control at most $f \leq\left\lfloor\frac{|\mathbb{X}|-1}{3}\right\rfloor$ group members whose collective reputation is less than $\frac{1}{3}$ of the cumulative reputation of the members of consensus group $\mathbb{X}$. Under this assumption, the system is safe and live.

The coverage of this assumption, i.e., how to constrain the adversary's ability of infiltrating the consensus group, to meet the aforementioned assumption, is explained in Section 4.3. The dynamics of the reputation mechanism allow the consensus group controlling RepuCoin to be infiltrated with safety, making it practically infeasible for an attacker to break the system (as shown in Table 2 of Section 6).

\section{RepuCoin}

In this section, we present details describing the different concepts and modules underlying RepuCoin. In particular, Section 4.1 details the different types of blocks, the leader election mechanism, and the reward system proposed. Then, we present our reputation-based weighted voting mechanism in Section 4.2, and the reputation system in Section 4.3.

\subsection{Block Mining and Reward System}

As mentioned earlier, in order to support higher throughput rates, RepuCoin decouples leader election (keyblocks) from transaction serialization (microblocks).

Keyblock and leader election. Miners solve Bitcoinlike puzzles to create keyblocks, and receive rewards corresponding to keyblock creations. However, the keyblock creator is not necessarily the leader that commits transactions into microblocks. Rather, the leader is randomly elected from the reputable miners. The puzzle is defined as follows:

$$
H(\text { prev_KB_hash } \| \text { Nonce } \| P K)<\text { target }
$$

where $H(\cdot)$ is a cryptographically secure hash function, prev_KB_hash is the hash value of the previous keyblock, $P K$ is the miner's public key, which the miner's reputation score $R$ is associated with, and target is a target value defined by the system. (For simplicity, we use reputation score and reputation interchangeably in this paper.)

RepuCoin solves forked chains on the fly by dynamically forming the consensus group and agreeing on which chain to choose. The consensus group members are the top reputed miners in the mining network. The reputation score of miners can be calculated by using data from the blockchain, and is maintained locally by each miner. When different miners have the same reputation, a naive solution would be to order them according to their public key $P K$ (a.k.a. address). However, this gives a miner with small $P K$ an advantage. To avoid this, in RepuCoin, miners with the same reputation score are ordered by $H(P K, R)$, where reputation $R$ (and therefore the hash value) is updated each time a new keyblock becomes part of the blockchain.

Each time a new keyblock is created, the creator proposes it to the consensus group. The group verifies the received keyblocks, and runs the underlying Byzantine agreement protocol to decide which keyblock to choose (if multiple conflicting keyblocks are proposed). We call a keyblock that is agreed upon and signed by the group a pinned keyblock. A pinned keyblock is final and canonical, it defines the unique global blockchain from the genesis block up to the pinned 
keyblock. All keyblocks that conflict with a pinned keyblock are considered invalid. New keyblocks are mined based on the hash value of the previous pinned keyblock.

The format of a pinned keyblock is as follows:

$\overbrace{\text { prev_KB_hash } \mid \text { Nonce } \mid \text { PK } \mid \text { R } \mid \text { K_sig } \mid \text { sig_KB_agmnt }}^{\text {keyblock }} \overbrace{\text { keyblock pin }}^{\text {sig }}$

The (new) KB_hash is the hash of a pinned keyblock, i.e., all the material in the frame above, where $K_{-}$sig is a signature on the hash value of (prev_KB_hash, Nonce, $P K, R$ ), and sig_KB_agmnt is the signed agreement from the consensus protocol on committing this keyblock. The first keyblock is called the genesis block (as in Bitcoin), which is defined as part of the system. Note that to verify a keyblock, consensus group members check the validity of $K_{-}$sig, the solution to the mining puzzle, and the reputation $R$.

Each time a new keyblock is pinned, the next leader - which verifies transactions and commits them into microblocks - is selected as follows:

$$
l_{i}:=x_{j} \quad \text { s.t. } x_{j} \in \mathbb{X} \wedge j=H\left(K_{-} \text {sig }_{i}\right) \bmod |\mathbb{X}|
$$

where $l_{i}$ is the $i$-th leader determined by the hash value $H\left(K_{-} s_{i} g_{i}\right)$ of the signature $K_{-} s_{i g}$ contained in the $i$-th pinned keyblock, and $\mathbb{X}$ is the set of miners constituting the consensus group. Since a cryptographically secure hash function is considered a random oracle, the leader is selected randomly in the consensus group with probability $\frac{1}{|\mathbb{X}|}$. However, one concern with this leader selection process is that consensus group members can determine the following leader before pinning a block. Thus, a consensus member interested in getting more rewards would only accept (decide to pin) a block that makes itself the new leader. To address this issue, a simple approach is to determine a leader by using $H\left(s_{i} g_{i}\right)$ instead of $H\left(K_{-} s_{i} g_{i}\right)$, where $s i g_{i}$ is a signature on the current length of the blockchain issued by the keyblock creator. Note that it is important to issue this signature only after the keyblock has been pinned by the consensus group. This way, each consensus member would accept a block with equal probability.

Remark 1. Note that in consensus schemes, a sufficient number of signed votes determines an agreement, and only one valid agreement can be reached. However, different combinations of (a sufficient number of) signed votes can be used to form this agreement. So, even though the agreement is unique, the collection (i.e., sig_KB_agmnt) of signed votes may have different valid values. This results in different valid prev_KB_hash on the same keyblock.

Hence, miners may solve their puzzles based on different valid prev_KB_hash. However, this will not form a fork in the system, as only one of the valid solutions to the puzzle will be validated by the consensus group, as detailed in $\S 4.2$. In addition, upon reaching the agreement on the next keyblock, the prev_KB_hash is considered the only valid hash value of the previous pinned keyblock, and only this value will be recorded in the blockchain.

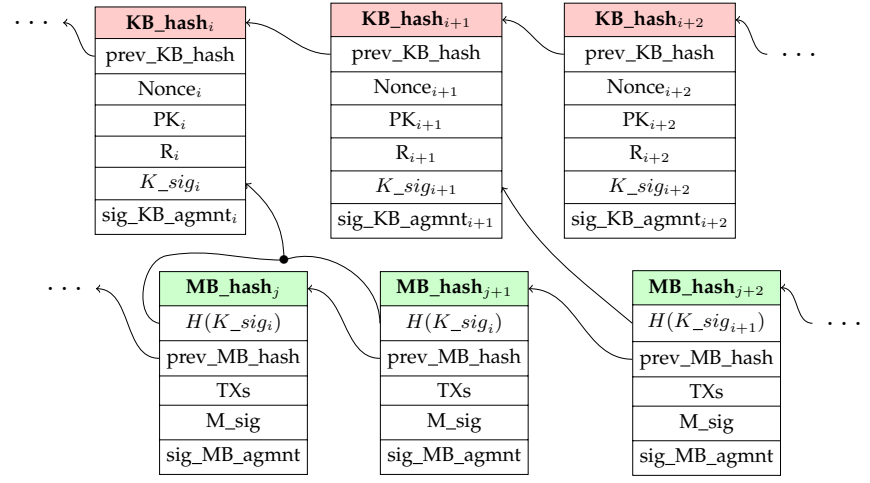

Fig. 1: A figure presentation of the blockchain structure.

Microblock. The current leader commits transactions into microblocks. To prevent double spending, each microblock is proposed to the consensus group before being accepted, and hence committing the transactions it encompasses. The group members verify the microblock, and initiate a consensus instance to agree on that microblock, which upon agreement is called a pinned microblock.

The format of a pinned microblock is shown below:

$$
\overbrace{\mathrm{H}(\text { K_sig) } \mid \text { prev_MB_hash } \mid \text { TXs } \mid \text { M_sig } \mid \text { sig_MB_agmnt }}^{\text {microblock }}
$$

where $H\left(K_{-}\right.$sig $)$is the hash value of the $K_{-}$sig contained in the current pinned keyblock, prev_MB_hash is the hash value of the previous pinned microblock; $T X s$ is a set of transactions organized as a Merkle tree, $M_{-}$sig is a signature on the hash value of (KB_hash, prev_MB_hash, $T X s)$, and sig_MB_agmnt is the signed agreement from the consensus protocol on the microblock. In this sense, in order to verify a microblock, consensus group members check the validity of $M_{-}$sig, verify the hash values of the keyblock and the previous microblock, and verify the set of transactions $T X s$. If invalid transactions are detected, then the leader is punished, as presented in $\S 4.3$. The (new) $M B \_$hash is the hash of the microblock (without a pin). In other words, the 'microblock pin' presented in the above frame is not part of the hash function's input. In this way, a leader can issue microblocks without waiting for the agreement, which optimizes the throughput of RepuCoin. The blockchain structure containing both keyblocks and microblocks is presented in Figure 1.

Reward system. In RepuCoin there are two types of rewards, namely mining rewards and transaction fees. Upon successfully mining a keyblock, a miner is entitled to get a reward, precisely if that miner's keyblock gets pinned. This mining reward is of a pre-set amount.

Every transaction within the microblock carries a transaction fee. The randomly elected leader shares the transaction fees with the miner of the pinned keyblock according to Algorithm 1. Roughly speaking, the miner's reputation determines the number of microblocks from which it can obtain transaction fees; the leader gets the rest. However, a leader that can determine the microblocks from which it gets transaction fees, may optimize its income by putting transactions with higher transaction fees into these microblocks. 
To avoid this unfair game, RepuCoin uses the hash value of the next pinned keyblock, i.e., the keyblock pinned at the end of the new epoch, to decide which pinned microblocks are allocated to the miner and which go to the leader. Since the hash value of the next pinned keyblock cannot be predicted, RepuCoin eliminates the above situation.

More precisely, let $\mathbb{M}=\left\{m_{0}, m_{1}, \ldots, m_{n-1}\right\}$ be the sequence of $n$ microblocks that are pinned by the consensus group. Let $R \in[0,1]$ be the reputation score of the miner which creates the $(i-1)$-th pinned keyblock. The transaction fees contained in the set $\mathbb{M}^{\prime}$ and $\mathbb{M}^{\prime \prime}$ of microblocks are shared between the miner of the $(i-1)$-th pinned keyblock and the leader, respectively, as shown in Algorithm 1.

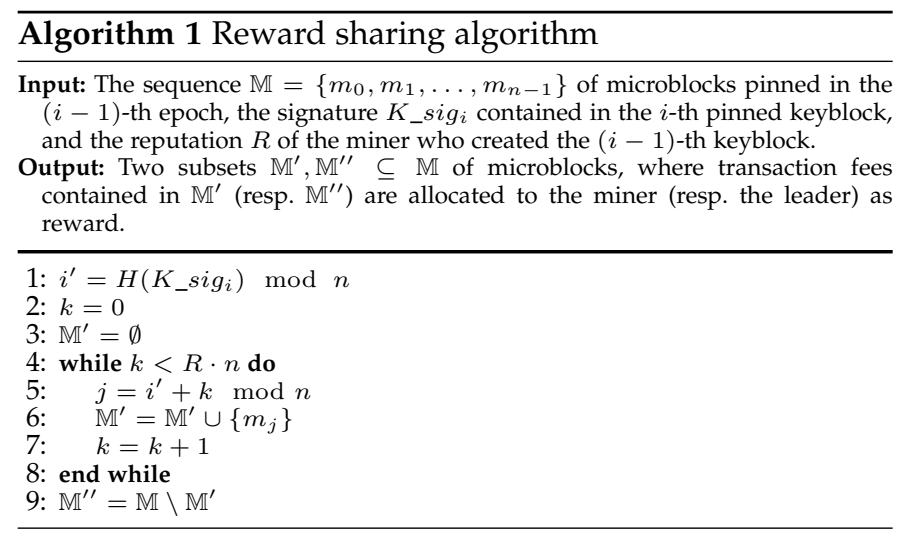

The way transaction fees are shared between keyblock creators and leaders motivates miners to increase their reputation. First, keyblock creators with higher reputation gain higher shares of rewards. Second, highly reputed miners constitute the consensus group; hence they can become leaders and get shares of transaction fees.

Similar to the Bitcoin system, to spend a reward, the miner simply makes transactions by using the $S K$ which is associated with the $P K$ contained in the keyblock, and provides the hash of the keyblock or microblock as an input of the transaction.

In Bitcoin, a miner needs to wait a maturity period of 100 blocks to avoid non-mergeable transactions from forks. In RepuCoin, each pinned keyblock and its underlying pinned microblocks are canonical, so leaders do not need to wait for this period to avoid non-mergeable transactions.

Remark 2. With RepuCoin, miners, even the newly joined ones with initial reputation, will get much more reward than what they can get in Bitcoin. In particular, a miner with RepuCoin gains the same mining reward as with Bitcoin, and gains transaction fees that are at least 60 times as high as with Bitcoin. A detailed analysis can be found in our full report [20].

\subsection{Block Pinning}

In RepuCoin, we use consensus to pin both keyblocks and microblocks. Transactions belonging to pinned microblocks cannot be unrolled at a later point in time. In this section, we describe in more detail the underlying consensus mechanism we use to pin such blocks.

Byzantine fault-tolerant consensus algorithms typically rely on processes voting. A process needs to collect a quo- rum of votes on a given value/action for that value/action to be considered legal by the system. The size of the quorum is selected in a way that guarantees (i) safety of decisions, e.g., avoiding conflicting decisions, and (ii) liveness of the system, i.e., miners should be able to hear eventually a number of votes on some value/action from a quorum of miners. It has been shown that in systems, as soon as $\frac{1}{3}$ or more of the miners are compromised, an attacker can make the system inconsistent - an attacker can make different parts of the system decide differently [31]. In order to make our system robust to such attacks, we propose to modify the traditional nominal voting mechanism, i.e., hearing from a sufficient number (quorum) of miners, by requiring as well to hear from a sufficient number of miners such that their added reputation is above a defined threshold. Such a modification prevents an attacker from breaking the correctness of the system directly upon compromising any $\frac{1}{3}$ of the miners: it should compromise as well enough miners that their added reputation is at least $\frac{1}{3}$ of the total reputation of the consensus group. Details on how to adapt a chosen BFT protocol to RepuCoin are presented in $\S 4.4$.

Consensus in RepuCoin employs a novel reputation-based weighted voting mechanism, i.e., rather than treating each vote from consensus group members equally (e.g., as in classic Byzantine protocols), the weight of each vote becomes its reputation over the total reputation of the group. More precisely, let $\left\{x_{1}, \ldots, x_{|\mathbb{X}|}\right\}$ be the consensus group, and each member $x_{i}$ of the group has its reputation score $R_{i}$. The weight of $x_{i}$ 's vote is $\frac{R_{i}}{\sum_{i=1}^{|X|} R_{i}}$, for all possible $x_{i}$.

Instead of only waiting to hear from at least $2 f+1$ nominal members to validate a value or an action, it is also necessary that the collective reputation of those members is more than $\frac{2}{3}$ of the total reputation of the consensus group.

Remark 3. Weighted-voting [33] is a classic and well known concept. The novelty of our weighted-voting system comes from the way that this weight is defined. More precisely, the weight of a miner's vote is given by this miner's reputation, i.e., its 'integrated power'. In particular, it considers the quantity and regularity of contributions over the entire blockchain, and provides a model to punish misbehaved miners (see section 4.3). In other words, we constrain the evolution of reputation over time. Thus, unlike in traditional systems, it becomes significantly difficult for an attacker to reobtain enough voting power after a deviation, or to flash-build it like in flash attacks.

Consensus group. As mentioned in Section 3.2, the size $|\mathbb{X}|$ of the consensus group is not pre-determined, but rather calculated by meeting a target percentage ${ }^{2}$ of the overall decision power. We select the members of the consensus group based on our reputation system; namely, the $|\mathbb{X}|$ miners with the top reputations constitute the members.

We define an epoch as the period between any two successive keyblocks that become part of the blockchain. Every epoch possesses a leader, which is the miner that should

2. Similar to the parameters of other systems, such as the block size of Bitcoin or the window size of ByzCoin, the target percentage of the overall decision power is a system parameter that can be reconfigured if necessary. 
issue a maximum pre-specified amount of microblocks. In every epoch, the reputation of only one miner, the creator of that pinned keyblock, may gain an extra increase; the reputation of all other miners would only have a very minor change according to Algorithm 2, or drops to " 0 " if they lie (see Section 4.3). Accordingly, given that $f \leq\left\lfloor\frac{|\mathbb{X}|-1}{3}\right\rfloor$ can be malicious, the members of the consensus group in any two consecutive epochs can differ by at most $f$ members. This stability in the members of the consensus group of consecutive epochs ensures the safety of consensus decisions. Namely, at the beginning of a new epoch correct consensus group members are aware of all committed transactions and hence do not accept/validate any conflicting transactions proposed by the new leader.

Committing microblocks. The leader of the current epoch, issues transactions in the form of microblocks. After generating a microblock, the leader initiates a consensus instance for this microblock proposing an accept to commit that microblock. Other consensus members will propose to either accept or decline (by not accepting) this microblock, depending on the transactions contained within and of course their validity. In other words, if transactions within the micro-block are invalid then members should decline committing that microblock. The leader continues to issue microblocks that are proposed to the consensus group for validation and commitment, until a new leader is elected.

Committing keyblocks. Upon successfully mining a keyblock, the miner of that block sends that keyblock to all members of the consensus group.

Upon receiving a keyblock, this group member initiates a consensus instance proposing the received keyblock (first received keyblock in the case when many such keyblocks are received). As a result, the members of the consensus group decide on a single keyblock to be part of the blockchain. The miner of that block is termed as the "winner". The hash of the new keyblock output by the consensus decides which member of the consensus group becomes the leader of the current epoch as previously mentioned.

A member of the consensus group that successfully decides on the identity of the new leader stops validating microblocks relative to the previous leader. Afterwards, that member initiates a consensus instance to agree on the total set of committed microblocks. Consensus group members need to agree on the total set of microblocks, since a leader is selected from this group. A leader that does not know the total set of committed microblocks might propose microblocks that are in conflict with committed ones and accordingly lose its reputation, not out of maliciousness but simply out of lack of knowledge. To avoid this situation, every member after reaching a decision on the identity of the new leader submits to consensus the largest sequence number of microblocks that have been committed along with a verifiable proof of this claim. Namely we assume that all consensus algorithms we use are implemented using digital signatures. As such, having a sufficient number $N$ of signatures on a decision constitute a proof of its validity. We say the number $N$ of signatures is sufficient if $N \geq 2 f+1$ and if the total reputation of the miners issuing these $N$ signatures is more than $\frac{2}{3}$ of the total reputation of the group.

Upon reaching a decision on the new leader and on
TABLE 1: The notations.

\begin{tabular}{|c|c|}
\hline Notation & Explanation \\
\hline$L$ & the length of the current blockchain; \\
\hline$c$ & the size of a block chunk, pre-defined by the system; \\
\hline$t$ & $\begin{array}{l}t=\left\lceil\frac{L}{c}\right\rceil \text { is the number of block chunks contained in a blockchain } \\
\text { with length } L \text {; }\end{array}$ \\
\hline Ext & the optional external source of reputation for the miner; \\
\hline$H$ & $\begin{array}{l}\text { a binary presenting whether the miner is honest }\left({ }^{\prime \prime} 1 \text { " }\right) \text { or not } \\
\left({ }^{\prime \prime} 0^{\prime \prime}\right) \text {; }\end{array}$ \\
\hline$k_{i}$ & the number of keyblocks created by the miner in chunk $i$ \\
\hline$N_{l}$ & the number of times that the miner is elected as a leader; \\
\hline$m_{j}$ & $\begin{array}{l}\text { the number of valid microblocks created by the miner at the } j \text {-th } \\
\text { time it is the leader; }\end{array}$ \\
\hline$m$ & $\begin{array}{l}\text { the maximum number of microblocks that a leader is allowed to } \\
\text { create, as defined by the system. }\end{array}$ \\
\hline mean $_{i}$ & $\begin{array}{l}\text { the mean value of keyblocks (if } i=k \text { ) or microblocks (if } i=m \text { ) } \\
\text { created by a miner or a leader across all epochs in the blockchain, } \\
\text { respectively. }\end{array}$ \\
\hline$s_{i}$ & the standard deviation corresponding to $\operatorname{mean}_{i}$, for $i \in\{k, m\}$. \\
\hline$(a, \lambda)$ & reputation system parameters \\
\hline
\end{tabular}

the global set of committed microblocks, each consensus group member also sends a message to notify the winner, the current leader, and the newly elected leader of this result. A consensus group member waits to either hear from consensus or from a sufficient number $N^{\prime}$ of other group members about the identity of the new leader and the global set of committed microblocks, before it adopts that member as leader. We say $N^{\prime}$ is sufficient if the total reputation of the issuers of the $N^{\prime}$ signatures is more than $\frac{1}{3}$ of the total reputation of the group and if $N^{\prime} \geq f+1$. In that case, the current leader simply stops issuing microblocks and the new leader takes over proposing microblocks.

Optimizing agreement on committed microblocks. In order to have agreement on the set of committed microblocks without resorting to a consensus instance, we propose the following optimization. Due to the PoW, it is known that mining a keyblock successfully takes a certain time depending on the mining difficulty (e.g. on average 10 minutes in Bitcoin). If we assume that a leader issues microblocks to be committed at a pre-specified rate, then we can assume that on average a leader commits $m$ microblocks per epoch. Accordingly, all consensus group members do not validate or commit more than $m$ microblocks for any given leader. Upon reaching a decision on the identity of a new leader (as a result of having a new keyblock mined) a consensus group member only initiates consensus on the set of committed microblocks if it has not seen $m$ committed microblocks from the previous leader.

The benefits of fixing the number of microblocks (that a leader can commit) to $m$ microblocks per epoch extends beyond having a fast and efficient agreement on the set of committed microblocks. It can also be used to incentivise leaders not to hinder throughput, e.g., a malicious leader in the worst case might decide not to submit any microblocks to intentionally stall the throughput. However now, since a leader is expected to commit $m$ microblocks per epoch, leaders which cannot meet that constraint can be punished for example by decreasing their reputation and hence decreasing their chances of staying part of the consensus group and becoming leaders again.

\subsection{Reputation System}

This section describes our reputation system and the proofof-reputation. We first highlight the shortcomings of pre- 
vious systems. For example, a proof-of-work based system requires a miner to show that it has done some work in order to include its set of proposed transactions, and hence extend the chain. Thus, a miner that has a high computing power can join the system at any time and can play attacks. Similarly, in the proof-of-membership system [15], a miner has to show that it has created enough blocks recently to demonstrate its computing power, then it can issue microblocks and can gain power in the consensus protocol. Again, an attacker with higher computing power can join the system at any time and can break the system. However, with proof-of-reputation, in addition to creating enough recent keyblocks, a miner has to show that it has behaved honestly and created keyblocks regularly for a period of time before being able to launch any attacks on the system.

Given the blockchain, the reputation of any miner can be calculated at any point in time. Accordingly, each miner maintains its own copy of the reputation score of all miners, based on the globally agreed blockchain. We denote by $R$ the reputation of a miner, which can take values in $[0,1]$. $R$ is calculated according to Algorithm 2. The notations are defined in Table 1.

In particular, Ext $\in[0,1]$ is the (optional) external source reputation of the miner. For example, when Citybank joins RepuCoin, it may have a starting reputation that is higher than a random individual joiner. In RepuCoin, this is encoded by using $E x t^{3} . H \in\{0,1\}$ is the honesty of the miner, which is set to " 1 " for each new joiner, and is set to " 0 " if a miner has misbehaved ${ }^{4}$. A miner is said to misbehave if:

- it presents conflicting signed messages to other consensus group members; or

- it commits microblocks with conflicting transactions when the miner is elected as leader.

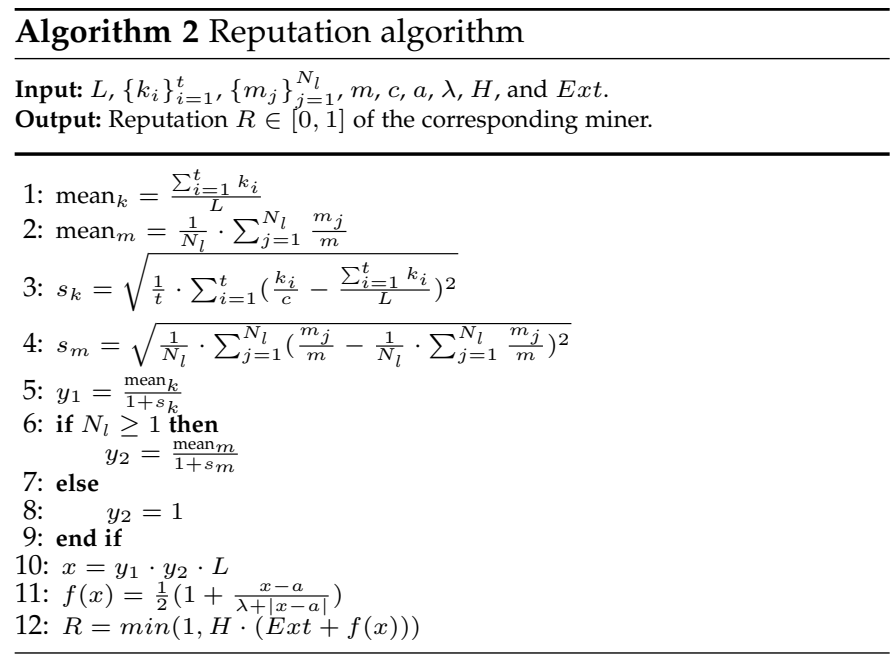

Upon their occurrence, an evidence of such misbehavior is included in the blockchain as a special transaction, similar to past work $[13,34]$. Non-Byzantine miners are incentivised

3. Note that this is only used to optimize the reputation system. However, to study the worst case, our analysis in $\S 6.3$ also shows the security guarantee without having this external source of reputation.

4. Once the honesty $H$ of a miner has been set to " 0 ", the $E x t$ of the corresponding entity will be set to " 0 " as this entity is not trustworthy. to place such a proof of fraud into the blockchain, to make malicious acts visible to everyone, hence preserving the health of the system. If a cryptocurrency system is not healthy, then its users will lose their confidence in the system. This may result in the plummeting of its currency exchange rate, and all miners will have a loss. So, miners are incentivised to keep the health of the system for their own profit.

The reputation function. We intended to define the social objectives of reputation in RepuCoin in a precise and parameterizable way. Those objectives are: (i) careful start, through an initial slow increase; (ii) potential for quick reward of mature participants, through fast increase in midlife; (iii) prevention of over-control, by slow increase near the top.

The formula defining the progression (resp. regression) of reputation, $f(x)$ above, is a sigmoid function. It ensures that miners, at the start, can only increase their reputation slowly, even if having a strong computing power. A miner needs to stay in the system and behave honestly for a long enough period, to progressively increase its reputation up to the turning point, where it is trusted enough to be incentivized to make it grow more quickly, to more interesting levels. And finally, the curve inflects again, so that the reputation does not grow forever, but asymptotically reaches a plateau that promotes a balance of power amongst miners. The reputation function is also parameterized, to allow to mark these points precisely, namely the parameters $(a, \lambda)$ can be tuned to adopt changes on when and how fast/slow miners can increase their reputation. The slope of $f(x)$ is directly correlated with the value of $\lambda$. The inflection point of $f(x)$ occurs at $x=a$, and is the point where a miner's reputation growth rate starts to decline.

We denote by a block chunk (or just 'chunk' for simplicity) a sequence of successive keyblocks in the blockchain. Blocks chunks satisfy the following: (i) all block chunks are of the same size, and (ii) any keyblock is included in exactly one block chunk.

$y_{1}$, defined at line 5 of Algorithm 2, captures the miner's "regularity" of generating keyblocks in each block chunk. In other words, $y_{1}$ shows how regularly the miner contributes its computing power to the system.

In particular, the numerator mean $k$ of $y_{1}$ is the percentage of pinned keyblocks generated by the miner, represents the fraction of valid work that a miner has contributed to the whole system. In the denominator, $s_{k}$ is the standard deviation of the pinned keyblocks generated by the miner, indicates the regularity with which a miner contributes to every chunk. Together, they guarantee that a miner's reputation is computed based on the miner's integrated power. Hence, a miner's integrated power is given by the total amount of valid work a miner has done over the period of time it has been active and the regularity of that work in the entire blockchain, rather than the miner's mining ability at a given time (or instantaneous power) as in classic proof-of-work. As such, when the system has been operated for some time, even a miner with strong computing power cannot build-up its reputation quickly: it needs to contribute honestly and regularly to the system to gain reputation. We present a more detailed analysis in $\S 6.3$.

Similarly, $y_{2}$ represents the "regularity" with which a 
leader commits the defined number of microblocks when it is selected. This incentivises leaders to optimize the throughput of RepuCoin.

\subsection{Adapting Existing BFT Protocols}

RepuCoin uses existing leader-based BFT protocols supporting digital signatures, to pin blocks. Apart from modifying the weight of the votes, RepuCoin also requires the following changes to adapt the existing BFT protocols.

Secure bootstrapping. With the potentially unbalanced amount of mining power in different blockchains, how to do secure bootstrapping is an open challenge in all blockchain systems. This, however, is not a problem with the classical BFT protocols, where the set of participants are predefined and fixed. Thus, to adapt existing BFT protocols, we need to provide a mechanism to establish a secure way to initialize consensus group, when no keyblock is created.

In RepuCoin, we assume the existence of a social community where participants vote to make decisions on several aspects of the system, such as the security parameters and external reputation factors. Such community exists for almost all permisionless blockchains. For example, with BitCoin this is the community who votes for proposals such as changing the maximum block size.

This community in RepuCoin votes a set of parties with external reputation to ensure a controlled bootstrapping, and record the result in the genesis block. The parties with initial higher external reputation will form the consensus group. During the secure bootstrapping phase, other miners with small or zero external reputation will gradually gain reputation and enter the consensus group.

View change. Classical BFT protocols provide view change - leader election and membership update - as a housekeeping function in the course of failures or recoveries in the (static) system participants roster. In addition to these technical functions, these protocols can be used in nonstandard ways in blockchain consensus. That was the case for example of ByzCoin, where a new leader is elected every time a new keyblock is created, by invoking the PBFT view-change protocol [15]. Similarly, with RepuCoin a view change is enforced by the pinning of a new keyblock, which ends an epoch, and thus establishes a consistent cut where the systems flushes (achieving consensus on the blockchain closing the epoch). Thus, several operations can be safely performed at this clean (re-)starting point: (i) a new consensus leader is elected; (ii) and the consensus membership is updated.

To adapt existing classical PBFT-like systems, the first difference is that, for (i), the leader election is deliberately provoked in this case (not on account of e.g., a failure) and the criterion changes to random selection, as presented in $\S 4.1$. For (ii), what happens is, again, a non-standard redefinition of the membership: the (ending epoch) consensus group re-evaluates the rule for consensus group formation (a quorum of the top reputed miners, see $\S 4.2$ ). Note that this can directly and deterministically be derived from the data in the blockchain, so consensus is safely achieved on the new roster of $\mathbb{X}$, which is installed for the new epoch. We recall that these operations occur through stable and safe states of the system, as mentioned before.
Crash/leave detection. As this is a permissionless environment, any miner can join, leave or crash at any time. If a consensus group member left the system, then RepuCoin will eventually detect that this has happened, by checking whether this member has been involved in the last instances of the consensus. If it is the epoch leader, view change ensues in the usual manner in BFT consensus protocols.

Message size. Most existing BFT protocols have been designed for state machine replication, and even optimized for short messages/commands. Performance shown in existing publications mostly concerns tests with relatively "small" block sizes. As shown in [35], the impact of largely increased block sizes on blockchain consensus performance should not be neglected either when choosing existing protocols, or when designing blockchain-specific BFT protocols.

\section{Performance Evaluation}

\subsection{Implementation}

Setup. We extend the BFT-SMaRt [36] library for our RepuCoin implementation. We deploy each member of the consensus group on a different machine, each having the following specifications: Dell FC430, Intel Xeon E5-2680 v3@2.5GHz, 48GB RAM. To simulate wide-area network conditions, we impose a round-trip network latency of 200 ms between any two machines, and a maximum communication bandwidth between any pair of machines to 35 Mbps. To better simulate the system in the real world scenario, we make use of the mining power distribution from the Bitcoin mining network ${ }^{5}$. More details and justifications of our setting, including the choice of PoW mining rate and mining power distribution, can be found in our technological report [20].

Consensus goup. We consider consensus groups that initially control from about $50 \%$ to $98.1 \%$ of computing power. With the current Bitcoin mining power distribution, the corresponding consensus group sizes would range from 4 to 19 . However, since we show, in $\S 6.3$, that security is hampered for consensus groups that control more than 90\% computing power, given Bitcoin's computing power distribution, we only present the performance results with consensus group controlling computing power from $44.7 \%$ to $90 \%$.

\subsection{Consensus Latency}

In this section, we measure the latency of our consensus implementation, and compare it with the latency of the original BFT-SMaRt. Such a comparison illustrates the timing overhead that is incurred relative to using our reputationbased weighted voting mechanism. We recall that in order to reach consensus, BFT-SMaRt requires at least $2 f+1$ members to agree on a value, while our reputation-based weighted voting variant requires in addition that these members (which are at least $2 f+1$ ) have collectively more than $\frac{2}{3}$ of the reputation of the entire consensus group.

5. The sequence of computing power of the top 24 pools is $(15.1,10.1$, 10.0, 9.5, 8.3, 7.1, 6.4, 5.9, 5.5, 4.0, 2.9, 2.8, 2.4, 2.2, 1.7, 1.5, 1.5, 0.7, 0.5, $0.5,0.3,0.3,0.2,0.2$ ), respectively. https://bitcoinchain.com/pools (as of April 2017) 


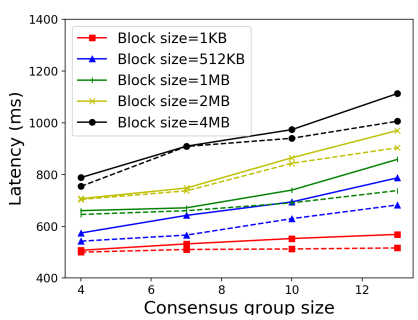

(a) Consensus latency

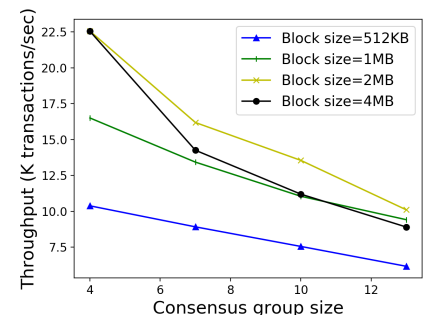

(b) Throughput.
Fig. 2: Performance evaluation, where (a) provides a comparison of consensus latency between BFT-SMaRt (dashed lines) and RepuCoin (straight lines); and (b) shows the throughput of RepuCoin.

We run experiments using keyblocks of size $1 \mathrm{~KB}$ and microblocks of sizes $512 \mathrm{~KB}, 1 \mathrm{MB}, 2 \mathrm{MB}$, and $4 \mathrm{MB}$. Unlike microblocks, keyblocks are typically small in size as they do not contain any transactions. Figure 2a presents the consensus latency of RepuCoin. It shows that RepuCoin and BFTSMaRt have a similar consensus latency values and patterns. For example, in both RepuCoin's consensus and BFT-SMaRt, consensus latency increases dramatically with the block size. The reason behind this trend can be explained by two things. First, it takes longer for the leader to propose microblocks to the consensus group, and for the group members to transmit a batch of the PROPOSE message which contains un-hashed microblock. Second, computing the hash value of a larger block and verifying the transactions it contains consume more time.

Moreover, in both RepuCoin's consensus and BFTSMaRt, when the group size increases from 4 (which controls $44.7 \%$ computing power of the network) to 13 (which controls $90 \%$ computing power of the network), the consensus latency increases by more than $50 \%$. However, despite this increase in latency, consensus can be reached in about 0.5-1.2 second, even when considering the blocks of size $4 \mathrm{MB}$.

\subsection{Throughput}

Figure $2 \mathrm{~b}$ presents the throughput of RepuCoin. First, as expected, our results in Figure $2 \mathrm{~b}$ show that the smaller the consensus group the higher the throughput. For example, using $2 \mathrm{MB}$ microblocks, the throughput increases from slightly more than 10000 TPS with a consensus group of size 13 (controlling $90 \%$ computing power), to 22500 TPS with a consensus group of size 4 (controlling $44.7 \%$ computing power). Second, for all group sizes, one can see, as expected, that the throughput tends to increase as blocks become larger, and this is what we observe up to 2MB. For example, when the consensus controls $90 \%$ computing power of the entire network (group size of 13), the throughput for blocks of $512 \mathrm{~KB}, 1 \mathrm{MB}$, and $2 \mathrm{MB}$, is respectively equal to 6200,9400 , and 10000 TPS. We observe that using larger block sizes (e.g., 4MB), decreases the throughput. However, this outlier is an artefact of the underlying protocol we use, i.e., the BFTSMaRt library, whose sheer performance, as a regular BFT protocol, is seemingly affected for very large block sizes, as discussed in Section 4.4.
When RepuCoin provides the best security guarantee (when the consensus group controls $90 \%$ computing power, as shown in §6.3), RepuCoin achieves a throughput of 10000 TPS when using $2 \mathrm{MB}$ blocks. This means that RepuCoin can handle the average transaction rates of Paypal and VISA as measured in real-life, which are 115 TPS and 1700 TPS respectively.

According to a survey [37], our throughput, i.e., 10K TPS, is outstanding among the analysed systems, as the reported peak figure for permissionless ledgers is also 10k TPS. We refer readers to the survey for more details.

\section{Security Analysis}

In this section, we present our analysis of the security provided by the mechanisms used in RepuCoin, namely reputation-based weighted voting consensus and proofof-reputation function. We begin by discussing the safety and liveness correctness conditions of the reputation-based weighted voting consensus protocol in Section 6.1, with predefined bounds on the relative reputation scores of participants. Then, we present in Section 6.2 a theorem (proven in [20]) showing that the achievement of those scores, which give decision power, is physically bounded by the conditions imposed on the growth rate of the proof-of-reputation function. Next, in Section 6.3, we present experiments exemplifying concrete values for the decision power growth vs. time in several situations, showing that RepuCoin indeed achieves very high stochastic robustness against attacks on its liveness or safety. Moreover, in Section 6.4 we provide an analysis of the non-rationality of infiltration attacks, with a comparison on the cost of attacking different systems. Finally, we describe in detail how RepuCoin prevents known attacks in Section 6.5.

\subsection{Reputation-based Consensus Safety and Liveness}

Unlike PoW-based systems, when using proof-ofreputation, an attacker cannot break the system by merely relying on its mining ability, i.e., its computing power. An attacker rather needs to gain reputation and hence contribute to the blockchain, by yielding pinned keyblocks. We recall that the reputation of a miner with correct behavior, in RepuCoin, builds essentially on its continued and regular contribution to the entire blockchain in addition to its external source of reputation Ext.

To study the worst case scenario, we do not consider any miner to have established an agreed upon trust, i.e. external reputation $E x t=0$ for all miners. For presentation simplicity we assume that every miner behaves honestly for some period of time that allows the attacker to have a sufficient reputation when intending to attack the system.

Let $\mathbb{X}=\left\{x_{1}, \ldots, x_{|\mathbb{X}|}\right\}$ be the consensus group. We note $R_{i}$ the reputation score of miner $x_{i}$, which gives it decision power. RepuCoin can rely on any underlying secure consensus algorithm, that can be adapted according to Section 4.4, to guarantee safety and liveness.

RepuCoin guarantees consensus safety, if: (i) the attacker controls no more than $f$ miners in the consensus group; 
or (ii) the consensus group members compromised by the attacker have a total reputation $R_{A}$ such that

$$
R_{A}<\frac{\sum_{i=1}^{|\mathbb{X}|} R_{i}}{3}
$$

where $R_{i}$ is calculated according to Algorithm 2. In other words, an attacker cannot break the safety unless both (i) and (ii) do not hold.

In addition, if any of the above two conditions does not hold, then an attacker can break the liveness of the system, i.e., an agreement may not be made on any block.

\subsection{Bounded Proof-of-Reputation Function Growth}

We hook the stochastic equations governing the evolution of our system to 'physics': it remains impossible to gain power faster than some upper bound derived from the need to perform a number of continued honest contributions to the network - what we called 'integrated power', to differentiate from 'instantaneous power', haunting all previous works by leading to flash attacks.

Our Theorem 1 (proven in [20]) shows that the proof-ofreputation function growth rate is bounded. In consequence, the rate of change of the decision power mentioned in the previous section is, at any time, limited.

Theorem 1. In RepuCoin, if the mining power of each participant remains the same, then at any time of the system, the rate of reputation increase of any node is bounded by $\frac{1}{2 \lambda}$, and the corresponding increase of the decision power of any consensus group member $i$ is bounded by $\frac{1}{2 \lambda} \Delta \operatorname{mean}_{k, i} \Delta L$.

Thus, with RepuCoin, the rate of increase of decision power in the entire system is limited regardless of the newly joined computing power. This makes RepuCoin secure against flash attacks launched by a late joiner, even when the attacker has a large amount of computing power. We refer readers to [20] for the full analysis and proofs.

\subsection{Proof-of-Reputation Attack Resilience}

So far, we have shown analytically that: RepuCoin is safe and live (Section 6.1) as long as decision power of attackers is below a defined threshold; then, we showed (Section 6.2) that it takes a known and bounded effort for attackers to reach that threshold and to control the consensus group. This section analyses how much would the attack effort be to reach the above-mentioned control.

Figure 3 and Table 2 show the requirements both in terms of the computing power and the time that should be spent doing honest work in the system, in order for an attacker to successfully launch any attack. In other words, they present the minimum effort to attack the liveness of RepuCoin.

Figure 3 indicates that the system is most secure when the consensus group $\mathbb{X}$ controls $90 \%$ computing power (with 13 nodes, i.e., $f=4$ ), and is most vulnerable when it controls $98.1 \%$ computing power. In fact, we can observe that the system (when $\mathbb{X}$ controls less than $90 \%$ computing power) becomes more secure as the consensus group controls more computing power of the network. After that

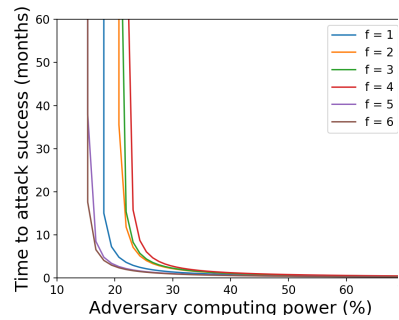

(a) Attack after 3 months

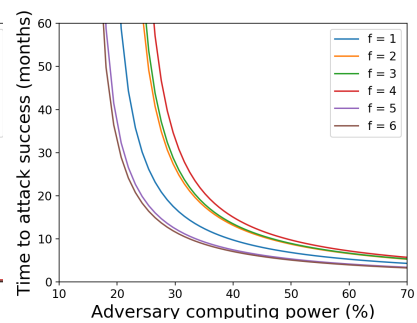

(b) Attack after 12 months
Fig. 3: Minimum effort to break the liveness of RepuCoin. We consider cases where an attacker joins after the system has operated for 3 months and a year, and where the consensus group controls mining power ranging from $44.7 \%$ (i.e., $\mathrm{f}=1,|\mathbb{X}|=4$ ) to $98.1 \%$ (i.e., $\mathrm{f}=6,|\mathbb{X}|=19$ ). The $\mathrm{x}$-axis shows the needed computing power and the $y$-axis shows the required time to attack the system.

increase the group size begins to depreciate the system security. These results can be explained by the fact that when the consensus group size grows beyond some point, the distribution of the computing power and reputation in the enlarged group could highly vary. For example, when $\mathbb{X}$ controls about $100 \%$ computing power of the system, more miners with relatively low reputation might become part of the consensus group; hence an attacker needs less time (and reputation) to infiltrate the consensus group and launch attacks. Our results, in Figure 3, show that if the consensus group controls $90 \%$ initial computing power, then an attacker joining after 3 months of system operation with $26 \%, 34 \%$, and $51 \%$ computing power of the entire network would need to work honestly for 22 months, 6 months, and 2.4 months respectively, to break the liveness of the system. If an attacker joins after 1 year, then it is infeasible for this attacker to break the system's liveness (and thus the system's safety) with a computing power $\leq 26 \%$; and the attacker would need 2 years (resp. 10 months) when possessing $34 \%$ (resp. $51 \%$ ) of the system's computing power. We say that it is infeasible for an attacker with $\leq 26 \%$ to successfully launch attacks, as our analysis shows that the attacker would have to contribute to the system honestly for 108 years before being able to do so. It is worth noting that an attacker with computing power $p_{a}$ joining a system whose computing power is $p_{s}$ would have $\frac{p_{a}}{p_{a}+p_{s}} \times 100 \%$ of the system's computing power.

In Table 2, we provide a different view on the attackers ability of successfully attacking the system's liveness. Breaking RepuCoin's safety is even harder than, at best as difficult as, breaking its liveness. It shows that an attacker who wants to break the system within one month after joining, i.e., by making the system lose liveness, would need to control at least $90 \%$ of the system's computing power, if that attacker joins after 3 months of the system being in operation. An attacker joining the system at a later time, e.g. 1 year (resp. 1.5 years) after the system operation, would never succeed in breaking the system's liveness nor safety within a period of 3 months after joining, and would require at least $68 \%$ (resp. 91\%) of the system's computing power to launch at attack within 6 months after joining. 
TABLE 2: The minimum computing power $(\mathrm{CP})$ and cost required to break the liveness of RepuCoin within a targeted time period, when an attacker joins the system at different times. sys : $* N$ means that the cost of attacking RepuCoin is at least $N$ times as high as the cost of attacking sys, where sys is either Bitcoin (BTC) or ByzCoin (BYZ).

\begin{tabular}{|c|c|c|c|c|}
\hline $\begin{array}{c}\text { Joining time } \\
\text { Target }\end{array}$ & 1 week & 1 month & 3 months & 6 months \\
\hline 1 month & infeasible & $\begin{array}{c}\text { CP: } 45 \% ; \\
\text { BTC: }{ }^{*} 635 ; \\
\text { BYZ: }{ }^{*} 6\end{array}$ & $\begin{array}{c}\text { CP: } 30 \% ; \\
\text { BTC: }{ }^{*} 1271 ; \\
\text { BYZ: }{ }^{*} 11\end{array}$ & $\begin{array}{c}\text { CP: } 27 \% ; \\
\text { BTC: }{ }^{*} 2287 \text {; } \\
\text { BYZ: }{ }^{*} 20\end{array}$ \\
\hline 3 months & infeasible & $\begin{array}{c}\text { CP: } 90 \% ; \\
\text { BTC: }{ }^{*} 1270 ; \\
\text { BYZ: }{ }^{*} 11\end{array}$ & $\begin{array}{c}\text { CP: } 45 \% ; \\
\text { BTC: }{ }^{*} 1906 ; \\
\text { BYZ: }{ }^{*} 17\end{array}$ & $\begin{array}{c}\text { CP: } 33 \% ; \\
\text { BTC: *2795; } \\
\text { BYZ: *25 }\end{array}$ \\
\hline 6 months & infeasible & infeasible & $\begin{array}{c}\text { CP: } 68 \% ; \\
\text { BTC: }{ }^{*} 2880 ; \\
\text { BYZ: }{ }^{*} 26\end{array}$ & $\begin{array}{c}\text { CP: } 45 \% ; \\
\text { BTC: }{ }^{*} 3812 ; \\
\text { BYZ: }{ }^{* 34}\end{array}$ \\
\hline 9 months & infeasible & infeasible & $\begin{array}{c}\text { CP: } 90 \% ; \\
\text { BTC: }{ }^{* 3812 ;} \\
\text { BYZ: }{ }^{* 34}\end{array}$ & $\begin{array}{c}\text { CP: } 54 \% ; \\
\text { BTC: }{ }^{*} 4574 ; \\
\text { BYZ: }{ }^{*} 41\end{array}$ \\
\hline 12 months & infeasible & infeasible & infeasible & $\begin{array}{c}\text { CP: } 68 \% ; \\
\text { BTC: *5760; } \\
\text { BYZ: *51 }\end{array}$ \\
\hline 18 months & infeasible & infeasible & infeasible & $\begin{array}{c}\text { CP: } 91 \% ; \\
\text { BTC: *7708; } \\
\text { BYZ: }{ }^{*} 69\end{array}$ \\
\hline 20 months & infeasible & infeasible & infeasible & infeasible \\
\hline
\end{tabular}

\subsection{Non-Rationality of Infiltration Attacks}

We hook heuristics to well-founded 'rationality': there is basically no rational economic model in RepuCoin that makes it worth to attack the network, i.e. it always costs much more than what would be gained, due to the following reasons.

First, attacks can only be successful after gaining enough reputation, by means of a lot of past investment and time spent (unlike all previous works, based on instantaneous power, a.k.a. computing power, which can be harnessed in several expeditious ways). Second, reputation goes to zero after the first detected attack (unlike all previous works, which essentially don't have memory and allow repeated attacks). Last but not least, the bribery attack by buying reputation is also made ineffective. More precisely, an adaptive adversary may try to acquire one or more nodes with high reputation, in order to trigger a 'flash reputation' attack. However, buying that reputation based power should cost at least as much as the investment previously made by the sellers, and the gain upon first use (and last, since reputation goes to zero), would never match the expense. So, it always costs much more than what would be gained.

To better illustrate the cost in different systems, Table 2 shows the cost of successfully attacking RepuCoin in comparison to the cost of attacking Bitcoin and ByzCoin, where the former is the most impactful system to date, and the latter is the only existing system where the voting power is also accumulated using mining power. For the analysis, we make use of the Bitcoin real-world mining power distribution, as presented in Section 5.1.

To successfully attack Bitcoin, in the best case (not considering the selfish mining attack), an attacker needs to have $51 \%$ of the computing power, and is required to maintain this computing power only for about an hour if 6 confirmations are required, to mine its own private chain on the side. Let $\alpha$ be the computing power (in unit) of the entire network, then the cost for each successful attack on
TABLE 3: Summary comparison of attack resilience

\begin{tabular}{l|c|c|c|c}
\hline Attacks & Bitcoin & Bitcoin-NG & ByzCoin & RepuCoin \\
\hline Flash attack & $\times$ & $\times$ & $\times$ & $\sqrt{ }$ \\
Selfish mining attack & $\times$ & $\times$ & $\times$ & $\sqrt{ }$ \\
Attack on consistency & $\times$ & $\times$ & $\times$ & $\sqrt{ }$ \\
Attack on liveness & $\sqrt{ }$ & $\sqrt{ }$ & $\sqrt{ }$ & $\sqrt{ }$ \\
Double spending attacks & $\times$ & $\times$ & $\sqrt{ }$ & \\
Eclipse attacks & $\times$ & $\times$ & \\
$\sqrt{ }$ - The system is secure against this attack. \\
$\times$ - The system is vulnerable to this attack. \\
1 The system is secure against eclipse attacks for double spending purpose, \\
however, if an attacker is able to partition the network, then it can \\
temporally delay the consensus process and reduce the throughput.
\end{tabular}

Bitcoin is about $0.51 \alpha r$, where $r$ is the price of maintaining 1 unit of computing power per hour.

With ByzCoin, in the best case (not considering the selfish mining attack), an attacker needs to have $34 \%$ computing power, and to maintain this power for the entire window (i.e. 1008 blocks), which is about a week. Thus, the cost is about $168 \cdot 0.34 \alpha r$, which is $57.12 \alpha r$. The cost of repeating this attack is the same, i.e., $57.12 \alpha r$.

With RepuCoin, in the worst case, where an attacker joins at the beginning of the system, RepuCoin does no better than Bitcoin and ByzCoin upon the first attack. However, to repeat the same attack, the attack would cost much more, as the reputation of the attacker would go to zero, and the attacker would be considered a late joiner.

For a later joined attacker, the minimum cost can also be calculated based on the required computing power, as shown in Table 2. For example, for a 6-month late joined attacker, to successfully attack the system within 3 months, the cost of attack is about $2160 \cdot 0.68 \alpha r$, which is about $1469 \alpha \mathrm{r}$. That is, the cost is 26 times as high as the cost of attacking ByzCoin, and 2880 times as high as the cost of attacking Bitcoin. Taking another example scenario, for a 1-year late joined attacker, it is infeasible to successfully attack the system within 3 months, even with the computing power of the entire network. However, the attacker is able to attack the system within 6 months with a cost of $4320 \cdot 0.68 \alpha r$, which is about $2938 \alpha r$. In this case, the cost is 51 times as high as the cost of attacking ByzCoin, and 5760 times of the cost of attacking Bitcoin.

\subsection{Defense Against Specific Attacks}

This section discusses defences of existing protocols against known attacks. Table 3 summarizes a comparison between Bitcoin, Bitcoin-NG, ByzCoin, and RepuCoin. More detailed discussions can be found in our report [20].

Flash attacks. In flash attacks [8], an attacker is able to obtain a temporary majority of computing power by renting enough mining capacity. This would break the security assumption of classic proof-of-work based systems.

RepuCoin, however, is resilient to flash attacks. As shown in $\S 6$, even an attacker with high computing power, depending on when that attacker joins, might require a very long period of time before being able to gain enough reputation to harm the system.

Selfish mining attack. In a selfish mining attack [6], an attacker keeps its mined blocks private, and publishes them according to some strategy that would allow the attacker 
to claim all rewards (with $>25 \%$ computing power).We refer readers to $[6,7,16]$ for more details on the attack, its generalization and its optimization.

RepuCoin pins each created keyblock, and new keyblocks can only be created based on the pinned keyblock. Given that RepuCoin relies on a reputation-based consensus and a secure signature scheme, no attacker can predict the hash value of a pinned block without controlling at least $2 f+1$ consensus group members that collectively have more than $\frac{2}{3}$ of the reputation of the entire consensus group. So, a selfish miner cannot gain any advantage in RepuCoin by hiding its created blocks.

Blockchain consistency and system liveness. Although Bitcoin-NG provides a high transaction throughput, it does not solve or address the consistency issues of Bitcoin. Namely, all transactions are only probabilistically valid. ByzCoin addresses these consistency issues providing deterministic transaction guarantees while achieving a high throughput. However, ByzCoin can permanently lose liveness during reconfiguration $[17,27]$, and a malicious miner can repeatedly make ByzCoin lose liveness temporarily [15].

RepuCoin provides strong transaction consistency and better liveness guarantees, as RepuCoin relies on a reputation-based Byzantine fault-tolerant consensus. Specifically, the consensus protocol in RepuCoin is deterministic for both keyblocks and microblocks.

Double spending attacks. RepuCoin addresses the double spending attack by speeding up the confirmation process to less than a few seconds, even when a block size is as large as $4 \mathrm{MB}$. In addition, RepuCoin provides a deterministic consistency guarantee rather than a probabilistic one. Determinism is achieved by pinning microblocks and keyblocks through a consensus scheme using a reputation-based voting mechanism. Pinned microblocks and keyblocks are nonreversible, a guarantee provided by our use of consensus.

Eclipse attacks and isolated leaders. In partial (or full) eclipse attacks $[4,5]$, an attacker capable of delaying information that a victim expects to receive is able to launch double spending attacks and selfish-mining attacks.

RepuCoin does not prevent an attacker from fully isolating a victim or delaying messages from a victim. However, given that blocks are pinned, the attacker cannot successfully launch double spending attacks, as previously explained. In the extreme case, some group members may be isolated temporally due to attacks on the network, e.g. those creating partitions. Such network attacks may delay the block pinning process and prevent RepuCoin from making progress. However, RepuCoin would recover as soon as the messages are delivered, and the attacker can neither create a fork of the blockchain, nor double spend any coin.

\section{Conclusion}

RepuCoin provides proof-of-reputation as an alternative way to provide a strong deterministic consensus, and be robust against attacks, in a permission-less distributed blockchain system. All BFT-based blockchain systems (e.g. $[15,17,38-40])$ are bound to the coverage of the assumption on the maximum number of faulty players, $f$, or their decision power quota thereof. RepuCoin, although belonging to that generation of systems, is the first to deploy effective mitigation measures that reduce brittleness in the face of overwhelming adversary power, where other systems give in. Namely, it provides security guarantees against an attacker who can control a majority of the overall computing power for a duration that increases with the joining time of the attacker.

Based on the strong deterministic guarantee derived from reputation-based weighted voting, the robustness of RepuCoin grows with legitimate operation time: the later the attacker joins, the more secure the system is. For example, an attacker that joins the system after it has been operating for a year, would need at least $51 \%$ of the overall computing power and would need to behave correctly in the system for 10 months before being able to successfully make RepuCoin lose liveness. Breaking RepuCoin's safety is at least as difficult as breaking its liveness. Further discussions on secure bootstrapping, formal security analysis, and applying our PoR to other virtual mining systems (e.g. proof of stake) can be found in our technological report [20].

\section{ACKNOWLEDGMENT}

This work is partially supported by the Fonds National de la Recherche Luxembourg (FNR) through PEARL grant FNR/P14/8149128.

\section{REFERENCES}

[1] Satoshi Nakamoto. Bitcoin: A Peer-to-Peer Electronic Cash System. 2009.

[2] Ghassan O. Karame, Elli Androulaki, and Srdjan Capkun. "Double-spending Fast Payments in Bitcoin". In: CCS. 2012.

[3] Maria Apostolaki, Aviv Zohar, and Laurent Vanbever. "Hijacking Bitcoin: Routing Attacks on Cryptocurrencies". In: IEEE SEP. 2017, pp. 375-392.

[4] Arthur Gervais et al. "Tampering with the Delivery of Blocks and Transactions in Bitcoin". In: CCS. 2015.

[5] Ethan Heilman et al. "Eclipse Attacks on Bitcoin's Peer-to-Peer Network". In: USENIX Security. 2015.

[6] Ittay Eyal and Emin Gün Sirer. "Majority Is Not Enough: Bitcoin Mining Is Vulnerable”. In: FC. 2014.

[7] Ayelet Sapirshtein, Yonatan Sompolinsky, and Aviv Zohar. "Optimal selfish mining strategies in Bitcoin". In: FC. 2016.

[8] Joseph Bonneau. "Why Buy When You Can Rent? Bribery Attacks on Bitcoin-Style Consensus". In: FC. 2016.

[9] Lightning Network. 2017. URL: https : / / lightning . network/.

[10] Joseph Poon and Thaddeus Dryja. The bitcoin lightning network: Scalable off-chain instant payments. 2015. URL: tinyurl.com/y9xoa42u.

[11] Kyle Croman et al. "On scaling decentralized blockchains". In: FC. 2016.

[12] Yonatan Sompolinsky and Aviv Zohar. "Secure highrate transaction processing in bitcoin". In: FC. 2015.

[13] Ittay Eyal et al. "Bitcoin-NG: A Scalable Blockchain Protocol". In: NSDI. 2016.

[14] J. Liu et al. "Scalable Byzantine Consensus via Hardware-assisted Secret Sharing". In: IEEE Transactions on Computers (2018). 
[15] Eleftherios Kokoris-Kogias et al. "Enhancing Bitcoin Security and Performance with Strong Consistency via Collective Signing". In: USENIX Security. 2016.

[16] Kartik Nayak et al. "Stubborn Mining: Generalizing Selfish Mining and Combining with an Eclipse Attack". In: IEEE SEP. 2016.

[17] Ittai Abraham et al. "Solidus: An Incentive-compatible Cryptocurrency Based on Permissionless Byzantine Consensus". In: CoRR abs/1612.02916 (2016).

[18] Rafael Pass and Elaine Shi. "Hybrid Consensus: Efficient Consensus in the Permissionless Model". In: DISC. 2017, 39:1-39:16.

[19] Jan Vermeulen. VisaNet handling 100,000 transactions per minute. MyBroadband. https: / / mybroadband.co. za / news / security / 190348-visanet-handling-100000transactions-per-minute.html. 2016.

[20] Jiangshan Yu et al. RepuCoin: Your Reputation is Your Power. Cryptology ePrint Archive, Report 2018/239. https:/ /eprint.iacr.org/2018/239. 2018.

[21] QuantumMechanic. Bitcoin Forum - Proof of Stake instead of Proof of Work. 2011. URL: https: / / bitcointalk. org/index.php?topic=27787.0.

[22] Andrew Miller et al. "Permacoin: Repurposing Bitcoin Work for Data Preservation". In: SEP. 2014.

[23] Sunoo Park et al. "Spacemint: A Cryptocurrency Based on Proofs of Space". In: IACR Cryptology ePrint Archive (2015).

[24] Sunny King and Scott Nadal. "Ppcoin: Peer-to-peer crypto-currency with proof-of-stake". In: self-published paper, August 19 (2012).

[25] Iddo Bentov et al. "Proof of Activity: Extending Bitcoin's Proof of Work via Proof of Stake". In: ACM SIGMETRICS Performance Evaluation Review 42.3 (2014), pp. 34-37.

[26] Intel. Proof of elapsed time (PoET). 2016.

[27] Philipp Jovanovic. ByzCoin: Securely Scaling Blockchains. 2016.

[28] Miguel Castro and Barbara Liskov. "Practical byzantine fault tolerance and proactive recovery". In: ACM Trans. Comput. Syst. 20.4 (2002), pp. 398-461.

[29] Giuliana Santos Veronese et al. "Efficient Byzantine Fault-Tolerance". In: IEEE Trans. Computers 62.1 (2013), pp. 16-30.

[30] T. Distler, C. Cachin, and R. Kapitza. "ResourceEfficient Byzantine Fault Tolerance". In: IEEE Transactions on Computers 65.9 (2016), pp. 2807-2819.

[31] Dahlia Malkhi and Michael K. Reiter. "Byzantine Quorum Systems". In: Theory of Computing. 1997.

[32] Cynthia Dwork, Nancy Lynch, and Larry Stockmeyer. "Consensus in the presence of partial synchrony". In: J.ACM. 1985.

[33] David K. Gifford. "Weighted Voting for Replicated Data". In: SOSP. 1979.

[34] Adam Back et al. Enabling blockchain innovations with pegged sidechains. 2014. URL: tinyurl.com/mj656p7.

[35] João Sousa, Alysson Bessani, and Marko Vukolic. "A Byzantine Fault-Tolerant Ordering Service for the Hyperledger Fabric Blockchain Platform". In: 48th Annual IEEE/IFIP International Conference on Dependable Systems and Networks, DSN 2018, Luxembourg City, Luxembourg, June 25-28, 2018. 2018, pp. 51-58.
[36] Alysson Neves Bessani, João Sousa, and Eduardo Adílio Pelinson Alchieri. "State Machine Replication for the Masses with BFT-SMART". In: DSN. 2014.

[37] Shehar Bano et al. "Consensus in the age of blockchains". In: arXiv preprint arXiv:1711.03936 (2017).

[38] Andrew Miller et al. "The Honey Badger of BFT Protocols". In: CCS. 2016.

[39] Yossi Gilad et al. "Algorand: Scaling Byzantine Agreements for Cryptocurrencies". In: SOSP 2017. 2017, pp. 51-68.

[40] Eleftherios Kokoris-Kogias et al. "OmniLedger: A Secure, Scale-Out, Decentralized Ledger". In: IEEE Symposium on Security and Privacy (2018).

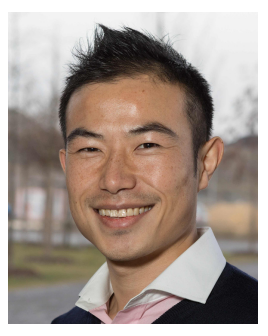

Jiangshan Yu is currently a lecturer (equivalent to U.S. assistant professor) at Monash Unviersity, Australia, and an honorary research fellow at the University of Birmingham, where he received his Ph.D in computer science in 2016. Previously, he was a research associate at SnT, University of Luxembourg (2017). The focus of his research has been on design and analysis of cryptographic protocols, cryptographic key management, blockchain consensus, and ledgerbased applications.

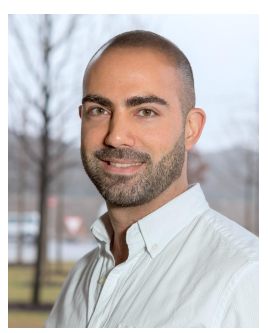

David Kozhaya is a Research Scientist at ABB Corporate Research, Switzerland. He received his $\mathrm{PhD}$ in Computer Science in 2016 from EPFL, Switzerland. His research interests include reliable distributed computing, real-time distributed systems, and fault- and intrusiontolerant distributed algorithms. His past work experiences span across interdisciplinary domains ranging from research, teaching, financial and market analysis, in addition to the management of various non-profit organizations.

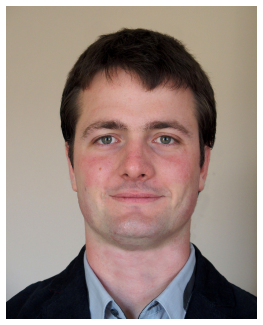

Jeremie Decouchant is currently a research associate at SnT, University of Luxembourg. He received his $\mathrm{Ph} . \mathrm{D}$ in computer science from the University of Grenoble-Alpes, France. Before that he obtained an engineering degree (MSc) from the Ensimag engineering school, in Grenoble. His research focused on the design and analysis of mechanisms to protect distributed systems against selfish or Byzantine faults. More recently, he studied how to rely on distributed systems and privacy principles to improve the

genomics workflows.

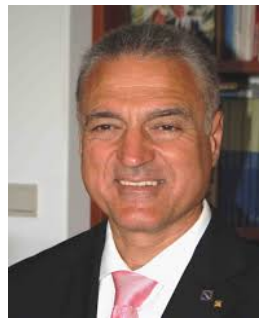

Paulo Esteves-Verissimo (Fellow, IEEE; Fellow, ACM) is currently a Professor and FNR PEARL Chair at the SnT and FSTC, University of Luxembourg (UL), and head of the CritiX group (http://wwwen.uni.lu/snt). He interests span secure and dependable distributed architectures, middleware and algorithms. Verssimo is Chair of the IFIP Working Group 10.4 on Dependable Computing and Fault-Tolerance and Vice-Chair of the Steering Committee of the IEEE/IFIP Dependable Systems and Networks (DSN) Conference. $\mathrm{He}$ is an Associate Editor of the IEEE Transactions on Computers. $\mathrm{He}$ is the author of over 180 peer-refereed publications and co-author of five books. 